\title{
Searching of a Different Approach for Target Therapy of Papillary Thyroid Cancer
}

\author{
GM Artykbaeva ${ }^{1 *}$ and NA Alimjanov ${ }^{2}$ \\ ${ }^{1}$ Institute of Bioorganic Chemistry Academy of Sciences, Uzbekistan \\ ${ }^{2}$ The Center for the Scientific and Clinical Study of Endocrinology, Ministry of Health, Uzbekistan \\ *Corresponding author: GM Artykbaeva, Institute of Bioorganic Chemistry Academy of Sciences, Tashkent, Uzbekistan
}

Submission: 韭 December 16, 2017; Published: 海 March 21, 2018

\begin{abstract}
Among thyroid cancer papillary thyroid carcinoma is the most widely met. As it was noted below papillary carcinoma covered 90 per cent of incidents of differentiated thyroid cancer. The loss of thyroid-specific functions during tumor progression determines the resistance of thyroid tumors to traditional radioiodine therapy. Specific drugs for aggressive and metastatic thyroid cancer are required for the development of effective therapy. Tyrosine kinase inhibitors (TKIs) are emerging as new tool for aggressive thyroid cancer. Over expression of MET (mesenchymal epithelial transition) receptor is found in papillary thyroid carcinoma. We aimed to study the phosphorylation of MET in the tissue of papillary thyroid carcinoma and effect of a TKI genistein on this process. The thyroid homogenates were incubated with genistein (Sigma-Aldrich, Germany) and then the phosphorylated MET was determined by Phospho-MET ELISA Kit (Sigma-Aldrich, Germany). In our studies, genistein at the concentrations of 10 and $20 \mu \mathrm{M}$ reduced receptor phosphorylation by $25.1 \%$ and $18.3 \%$, respectively. The native serum of the patient with papillary carcinoma increased phosphorylation of MET by $44.6 \%$. Our results provide data that genistein suppresses phosphorylation of MET in tissue of thyroid cancer in physiological doses. The effect of genistein on MET receptor, responsible for mesenchymal-epithelial transition, indicates its anti-metastatic properties.
\end{abstract}

Keywords: Thyroid Carcinoma, Tyrosine Kinase, MET Receptor, Phosphorylation, Genistein.

Abbreviations

TKIs: Tyrosine Kinase Inhibitors; MET: Mesenchymal Epithelial Transition; HGF: Hepatocyte Growth Factor; DTC: Differentiated Thyroid Carcinoma; PTC: Papillary Thyroid Cancer; FTC: Follicular Thyroid

\section{Introduction}

Differentiated thyroid carcinomas (DTCs) that arise from follicular cells are separated into two types-papillary thyroid cancer (PTC) (90\%) and follicular thyroid cancer (FTC) (10\%). Complete total thyroid ectomy is the treatment of choice for PTC and FTC. Radioiodine is routinely recommended in high-risk patients and considered in intermediate risk DTC patients. Thyroid cancer cells may lose their iodide uptake ability and become resistant to radioiodine. The lack of specific medicines for aggressive and metastatic DTC has leads to the needs for the development of new drugs [1].

Tyrosine kinase inhibitors (TKIs) are small organic molecules inhibiting tyrosine kinase auto-phosphorylation; most of them are multi-kinase inhibitors. TKIs act on the molecular pathways involved in growth, angiogenesis, local, and distant spread of TC. TKIs are emerging as new therapies of aggressive TC, being capable of inducing clinical responses and stabilization of disease. New efforts are made to find new more effective and safe compounds and to personalize therapy in each thyroid cancer patient [2].
Overexpression of MET (mesenchymal epithelial transition) receptor is found in papillary thyroid carcinoma. Abnormal activation of MET is responsible for obtaining a tumorigenic phenotype with metastases. Activated by the growth factor of hepatocytes (HGF), MET receptor can be used as a therapeutic target for the treatment of cancer and metastases [3]. We aimed to study the phosphorylation of MET in the tissue of papillary thyroid carcinoma and effect of TKI genistein on this process. So far therapy with thyroid cancer targeted drugs remained outside the field of view of researchers due to the lack of drugs with recorded indications for thyroid cancer.

\section{Materials and Methods}

From the group of patients with various thyroid cancers, 8 tissue samples of female patients of the Center for the Scientific and Clinical Study of Endocrinology, Ministry of Health, Uzbekistan with a verified histological diagnosis of PTC at the age of 35 to 56years were selected. The median tumor diameter of papillary carcinomas was $2.5 \mathrm{~cm}$. The tissues of PTC were homogenized in a $0.25 \mathrm{M}$ 
sucrose buffer-0.001M EDTA pH 7.3 and then were incubated with genistein (Sigma -Aldrich, Germany) at the concentrations of 10 and $20 \mu \mathrm{M}$ for 1 hour at $37{ }^{\circ} \mathrm{C}$ in presence of $\mathrm{HGF}(30 \mathrm{ng} / \mathrm{ml})$. The phosphorylated MET level was determined by Phospho-MET ELISA Kit (Sigma-Aldrich, Germany).

\section{Statistical Analysis}

All data were processed by Microsoft Excel, STATISTICA 6 , and Biostat programs. Intergroup differences were considered significant at $\mathrm{P}<0.05$.

\section{Results}

We have determined the phosphorylation of MET receptor in the tissue of thyroid cancer. Tissue homogenates were incubated with genistein at the concentrations of 10 and $20 \mu \mathrm{M}$, and then the receptor phosphorylation was measured. As a positive control, the thyroid tissues were stimulated by blood serum of a patient with papillary thyroid carcinoma, which is characterized by increased amounts of growth factors, in particular, HGF. Another positive control used was exogenous HGF (Sigma, Germany). In our studies, genistein at the concentration of $10 \mu \mathrm{M}$ reduced receptor phosphorylation by $25.1 \%(\mathrm{P}<0.05)$. An increase of concentration of genistein twice $(20 \mu \mathrm{M})$ decreased phosphorylation by $18.3 \%$ $(\mathrm{P}<0.05)$. The serum of the patient with papillary carcinoma increased phosphorylation by $44.6 \%$ (Figure 1). The specific ligand of MET receptor-hepatocyte growth factor-also increased the phosphorylation of the receptor by $64 \%$. Our results provide data that genistein suppresses phosphorylation of MET in tissue of thyroid cancer in physiological doses.

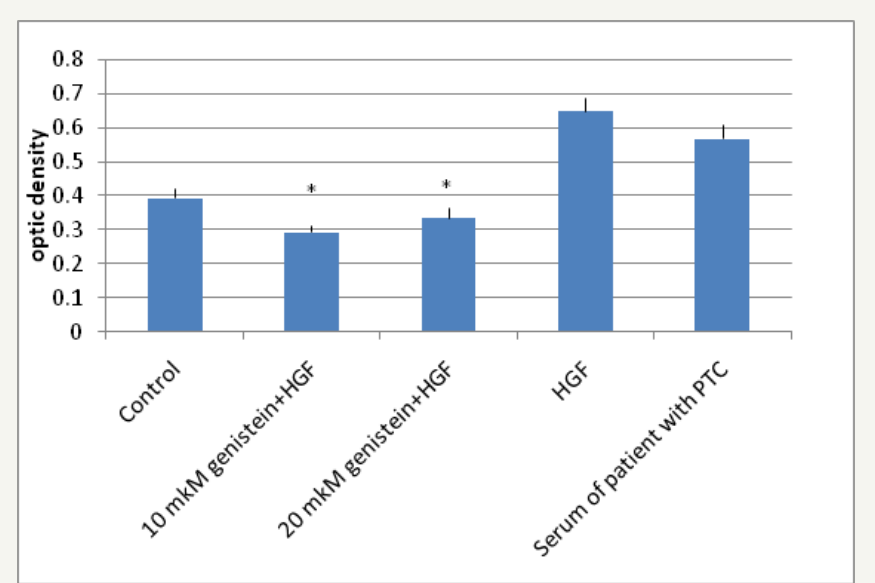

Figure 1: Phosphorylation of MET receptor in thyroid cancer tissue

${ }^{*} \mathrm{P}<0.05$-significance regarding control.

\section{Discussion}

For PTC, over expression of the tyrosine kinase receptor MET is characteristic, which is caused by increased secretion of growth factor HGF [4]. HGF is a potential mitogen for epithelial cells, follicular and C-cells of thyroid gland, promotes invasive growth of tumors. HGF and MET are involved in the regulation of cell migration. HGF and MET both are involved in postnatal physiological and patho-physiological processes. Their increased expression is found in some damaged tissues [5]. The disruption of the pathway of MET and its ligand serves as a signal of malignancy in humans. A large number of studies have shown that HGF and MET are often expressed in carcinomas and other solid tumors, their metastases, which correlates with a poor prognosis. Expression of MET oncogene is associated with aggressive biological behavior of the tumor and a high risk of metastasis $[6,7]$.

In normal thyroid cells, MET is not expressed, or is expressed at a very low level. In contrast, a high level of expression of this oncogene is more often a molecular abnormality in papillary thyroid cancer, including microadenomas. This indicates that the expression of MET is a frequent and early event in the carcinogenesis of the papillary thyroid cancer [8]. Activation of the receptor starts with the phosphorylation of the tyrosine kinase domain. The phosphorylated receptor triggers the underlying mitogen-activating cascade to generate a tumorigenic signal. A promising approach in the treatment of tumors is the blocking of this process by various agents. For this purpose, small molecular tyrosine kinase inhibitors are used that have the ability to bind to the adenosine triphosphate site of the receptor and interrupt transmission of the pathological signal. As a small molecular inhibitor, we used isoflavone genistein. Genistein exhibits affinity to the ATP-binding center of protein kinase by ATP-competitive manner and serves as a potent inhibitor of tyrosine kinase activity of growth factor receptors of transformed target cells [9]. It was shown previously that genistein inhibited tyrosine kinase activity only at the upper limit of the physiological dose $(\geq 10 \mu \mathrm{M})[10,11]$. The role of isoflavones has traditionally been associated with inhibition of proliferation and induction of apoptosis. Some works provides evidence that isoflavones are involved in cellular processes associated with tumorigenesis [12]. The effect of genistein on the MET receptor, responsible for mesenchymal-epithelial transition, indicates its anti-metastatic properties.

Further, overexpression of MET found in papillary carcinoma is associated with wild type of receptor and considered to be ligand-dependent since neither MET mutations nor the major structural changes in the MET protein in papillary carcinoma have been found [13]. We found no mutation in 19-21exons of MET responsible for tyrosine kinase domain [14]. Given the role of MET in the progression of cancer, in selecting a treatment strategy it is important to keep in mind the mechanism of this activation. In ligand-dependent activation, the search for ligands inhibiting tyrosine kinase receptors is relevant. Conversely, tyrosine kinase inhibitors will be ineffective if the protein is mutant and in the state of constant activity. Thus, for the receptors carrying specific mutations, expensive antibodies are efficiently synthesized. For wild type receptors TKIs would be appropriate for use.

\section{Conclusion}

We have studied in vitro inhibition of the initial stage of 
tumorigenic process in the postoperative tissue of thyroid cancer with a small molecular inhibitor of plant origin. Studies on this matter have been conducted on cell culture lines. In our study genistein reduced phosphorylation of MET in tissue of thyroid cancer in physiological doses. For MET associated with wild type receptor it is relevant to use small molecule inhibitors of kinase domain.

\section{References}

1. Garajjova I, Giovannetti E, Biasco G, Peters GJ (2015) c-MET as a target for personalazed therapy. Transl Oncogenomics 7 (Suppl 1):13-31.

2. Ferrari SM, Fallahi P, Politti U, Materazzi G, Baldini E, et al. (2015) Molecular targeted therapies of aggressive thyroid cancer. Front Endocrinol (Lausanne) 20(6): 176

3. Youles M, Holmes O, Petoukhov M, Nessen M (2008) Engineering the NK1 fragment of hepatocyte growth factor/scatter factor as a MET receptor antagonist. J Mol Biol 377(3): 616-622.

4. Fu YT, Zheng HB, Zhou L, Zhang DQ Liu XL, et al. (2017) Valproic acid, targets papillary thyroid cancer through inhibition of c-Met signalling pathway. Am J Transl Res 9(6): 3138-3147.

5. Migliore C, Giordano S (2008) Molecular cancer therapy: can our expectation be MET?. Eur J Cancer 44(5): 641-651.

6. Rocci A, Gambella M, Aschero S, Baldi I, Trusolino L, et al. (2014) MET dysregulation is a hallmark of aggressive disease in multiple myeloma patients. Br J Haematol 164(6): 841-850.
7. Belfiore A, Gangemi P, Costantino A, Russo G, Santonocito GM, et al. (1997) Negative/low expression of the MET/hepatocyte growth factor receptor identifies papillary thyroid carcinomas with high risk of distant metastasis. J Clin Endocrinol Metab 82(7): 2322-2328.

8. Klotz M, Schmid E, Steiner Hahn K, Rose T, Laube J, et al. (2012) Preclinical evaluation of biomarkers for response monitoring to the MET inhibitorBAY-853474. Biomarkers 17(4): 325-335.

9. Latocha M, Pjonka J, Kuemierz D, Jurzak M, Polaniak R, et al. (2014) Transcriptional activity of genes encoding MMPs and TIMPs in breast cancer cells treated by genistein and in normal cancer-associated fibroblasts in vitro studies. Acta Pol Pharm 71( 6): 1095-1102.

10. Mahmoud AM, Zhu T, Parray A, Siddique HR, Yang W, et al. (2013) Differential effects of genistein on prostate cancer cells depend on mutational status of the androgen receptor. PLoS One 8(10): e78479.

11. Wang S, DeGroff VL, Clinton SK (2003) Tomato and soy polyphenols reduce insulin-like growth factor-I-stimulated rat prostate cancer cell proliferation and apoptotic resistance in vitro via inhibition of intracellular signaling pathways involving tyrosine kinase. J Nutr 133(7): 2367-2376.

12. Mahmoud AM, Yang W, Bosland MC (2014) Soy isoflavones and prostate cancer: a review of molecular mechanisms. J Steroid Biochem Mol Biol 140: $116-132$.

13. Wasenius VM, Hemmer S, Karjalainen Lindsberg ML, Nupponen NN, Franssila K, et al. (2005) MET receptor tyrosine kinase sequence alterations in differentiated thyroid carcinoma. Am J Surg Pathol 29(4): 544549 .

14. Artykbaeva GM, Chechulina MV, Salikhov RS (2014) Study of structural alteration of MET gene by sequencing. J Theor Clin Med 2(3): 16-17.
Creative Commons Attribution 4.0

International License

For possible submissions Click Here

\section{Submit Article}

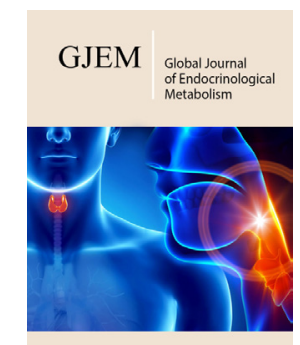

Global Journal of Endocrinological Metabolism

\section{Benefits of Publishing with us}

- High-level peer review and editorial services

- Freely accessible online immediately upon publication

- Authors retain the copyright to their work

- Licensing it under a Creative Commons license

- Visibility through different online platforms 